\title{
Lidil
}

Revue de linguistique et de didactique des langues

38 | 2008

Langue du droit

\section{Décrire et prescrire : l'harmonisation de la terminologie juridique multilingue}

Céline Randier et Leonhard Voltmer

\section{(2) OpenEdition}

\section{Journals}

Édition électronique

URL : http://journals.openedition.org/lidil/2777

DOI : $10.4000 /$ lidil.2777

ISSN : $1960-6052$

\section{Éditeur}

UGA Éditions/Université Grenoble Alpes

\section{Édition imprimée}

Date de publication : 1 décembre 2008

Pagination : 33-52

ISBN : $978-2-84310-130-4$

ISSN : 1146-6480

\section{Référence électronique}

Céline Randier et Leonhard Voltmer, « Décrire et prescrire : I'harmonisation de la terminologie juridique multilingue », Lidil [En ligne], 38 | 2008, mis en ligne le 01 juin 2010, consulté le 30 avril 2019. URL:

http://journals.openedition.org/lidil/2777; DOI : 10.4000/lidil.2777

Ce document a été généré automatiquement le 30 avril 2019.

(C) Lidil 


\title{
Décrire et prescrire : l'harmonisation de la terminologie juridique multilingue
}

\author{
Céline Randier et Leonhard Voltmer
}

Nous présentons ici le projet LexALP, précurseur dans le domaine de l'harmonisation juridique multilingue. L'objectif est de décrire, comparer et harmoniser la terminologie juridique dans les domaines clés de l'aménagement du territoire et du développement durable. La terminologie harmonisée servira de fondement à la communication internationale dans les Alpes. Pour atteindre cet objectif, le projet a développé tous les outils méthodologiques et informatiques nécessaires : un corpus de textes juridiques, un extracteur automatique de définitions, une méthode scientifique. Le caractère innovant du projet est situé principalement au niveau de la méthode, plus complexe que celle de la normalisation technique (Chiocchetti et Voltmer, 2008b). Les résultats de ce projet viennent d'être publiés ${ }^{1}$.

1 Après un bref rappel de la problématique de la normalisation et de l'harmonisation juridiques, nous décrivons dans la deuxième section les diverses étapes qui mènent de la législation multilingue à la terminologie harmonisée multilingue en mettant l'accent sur l'interdépendance de ces étapes. La troisième section utilise la polarité entre " description » et " prescription » comme clé d'interprétation pour mieux comprendre ce que comporte une activité complexe comme celle de l'harmonisation terminologique. Dans les conclusions, nous revenons sur le procédé d'harmonisation en mettant en valeur son utilité et ses implications pour d'éventuels projets futurs.

\section{Normalisation et harmonisation juridiques}

La normalisation et l'harmonisation ${ }^{2}$ de la terminologie juridique sont encore un travail peu commun, mais se développeront certainement dans l'avenir. Dans l'industrie et toutes les branches liées à la mondialisation, l'utilité et même la nécessité de la terminologie spécialisée plurilingue est désormais pleinement reconnue. Dans le domaine 
du droit, essentiellement dominé par des nations unifiées sur la base et à l'aide d'une seule langue nationale, le plurilinguisme accuse un retard par rapport au secteur économique. Actuellement, face aux besoins croissants, apportés par les différents accords supranationaux qui impactent les juridictions nationales, les normes plurilingues se développent jour après jour sur les plans international, européen et régional.

Dans les domaines des sciences et des technologies, et notamment la médecine, les efforts d'harmonisation ont souvent été couronnés de succès ${ }^{3}$. Cependant il n'existe pas encore de pendant à la normalisation pour le domaine juridique. L'Union européenne utilise une terminologie multilingue, mais elle n'a pas un seul concept qui possède des équivalents "sûrs " dans plusieurs langues. Des prescriptions dans le domaine de la traduction n'existent pas. Néanmoins la Communauté utilise une terminologie multilingue quotidiennement dans ses textes officiels, qui présentent le même contenu en plusieurs langues en parallèle. Cette terminologie juridique implicite a été regroupée dans une base de données terminologique accessible au public, la base de données IATE ${ }^{4}$. Mais il s'agit de données descriptives : toutes les variantes, une fois utilisées, sont présentées sans aucune ligne directrice indiquant le terme de préférence.

3 Prenons un exemple important, celui d'un des premiers traités de la Communauté, le «Traité instituant la Communauté européenne de l'énergie atomique ». Pour IATE, les deux synonymes français "traité CEEA» et «traité Euratom» sont fiables et corrects. Cette expression a été traduite plus tard dans toutes les autres langues officielles. On ne dénombre pas moins de quatre traductions en anglais. La logique voudrait une équivalence deux à deux entre les deux langues par une relation de traduction (en l'occurrence, le terme « Rome Treaty » n'a pas d'équivalent en français). Rien n'interdit ni ne suggère les préférences de termes: un terme français F1 peut être traduit par les termes anglais A1, A2 ou A3 indistinctement, tel des synonymes, sans aucune normalisation. Par ailleurs, aucune justification n'est donnée pour le critère de validité des termes. Deux des trois expressions anglaises sont « très fiables » alors que la troisième est seulement «fiable», bien que tous les trois soient synonymes, et désignent par définition le même concept.

4 À la différence de LexALP, la banque de données IATE reste exclusivement descriptive, sans lignes directrices ni sur le terme de préférence dans une langue, ni sur l'équivalence de termes entre les diverses langues. En principe, la Communauté est compétente pour réglementer son langage, codifier son propre mode d'expression. Quant à l'utilité de la normalisation, nous pouvons donner l'exemple de la traduction du mot allemand Kohle ou anglais coal : IATE donne les deux termes « charbon » et « houille ", sans définition. Même pour la relation entre les langues avec deux termes, comme le français et l'italien (carbone et carbone fossile), il n'est pas possible de déterminer le terme correspondant.

5 Le besoin même de traduire la législation européenne montre qu'il n'y a pas de rédaction en commun (co-drafting), que le texte ayant valeur juridique dans les 23 langues officielles ne nait pas en même temps dans ces 23 langues $^{5}$. Il s'agit plutôt d'une forme d'autotraduction ou de multilinguisme d'écriture ${ }^{6}$.

6 En pratique, le droit européen se fonde sur les grands systèmes juridiques de l'Union européenne. Les concepts juridiques sont alors difficiles à traduire car empruntés à des langues et systèmes éloignées de la langue juridique d'arrivée. Au lieu d'une simple activité de traduction, la normalisation doit faciliter la compréhension en rapprochant dès le départ les concepts utilisés. L'introduction de relations de traduction fiables 
permet aussi une retraduction plus efficace, par exemple dans le cas d'une procédure contentieuse devant la Cour de Justice. Il s'agit ici d'un processus qui est bien connu et documenté pour la normalisation technique, mais qui est presque nouveau pour la terminologie juridique (Heutger, 2004).

\section{Un travail d'harmonisation fondé sur le corpus}

Le projet LexALP se positionne là où IATE s'arrête. Le processus d'harmonisation démarre à partir des textes dans les quatre langues de la Convention alpine. Des interprètestraducteurs ${ }^{7}$, des juristes et des linguistes ont choisi mille concepts considérés comme les plus importants pour les quatre langues. La subdivision des termes en différents domaines suit celle de la Convention alpine en différents protocoles thématiques. Ensuite les termes choisis ont été regroupés avec leurs expressions correspondantes dans les trois autres langues, identifiés par une recherche dans le corpus parallèle des textes de la Convention alpine. Dans cette phase, les termes risquent de se multiplier, parce que la recherche d'un seul terme A dans les textes parallèles peut extraire deux termes B1 et B2 dans une autre langue. La recherche avec B1 et B2 peut extraire $\mathrm{C} 1, \mathrm{C} 2, \mathrm{C} 3$ et $\mathrm{C} 4$ dans la troisième langue. Ensuite il se peut même que la retraduction des termes $\mathrm{B} 1$ et $\mathrm{B} 2$ renvoie non seulement en A, mais aussi à une ou plusieurs autres expressions. À ce point nous sommes déjà pleinement dans la terminologie, parce que nous souhaitons bien sûr rester dans le signifié d'un seul et même concept. Ceci est une des raisons pour lesquelles la tentative d'extraction automatique initialement envisagée a échoué.

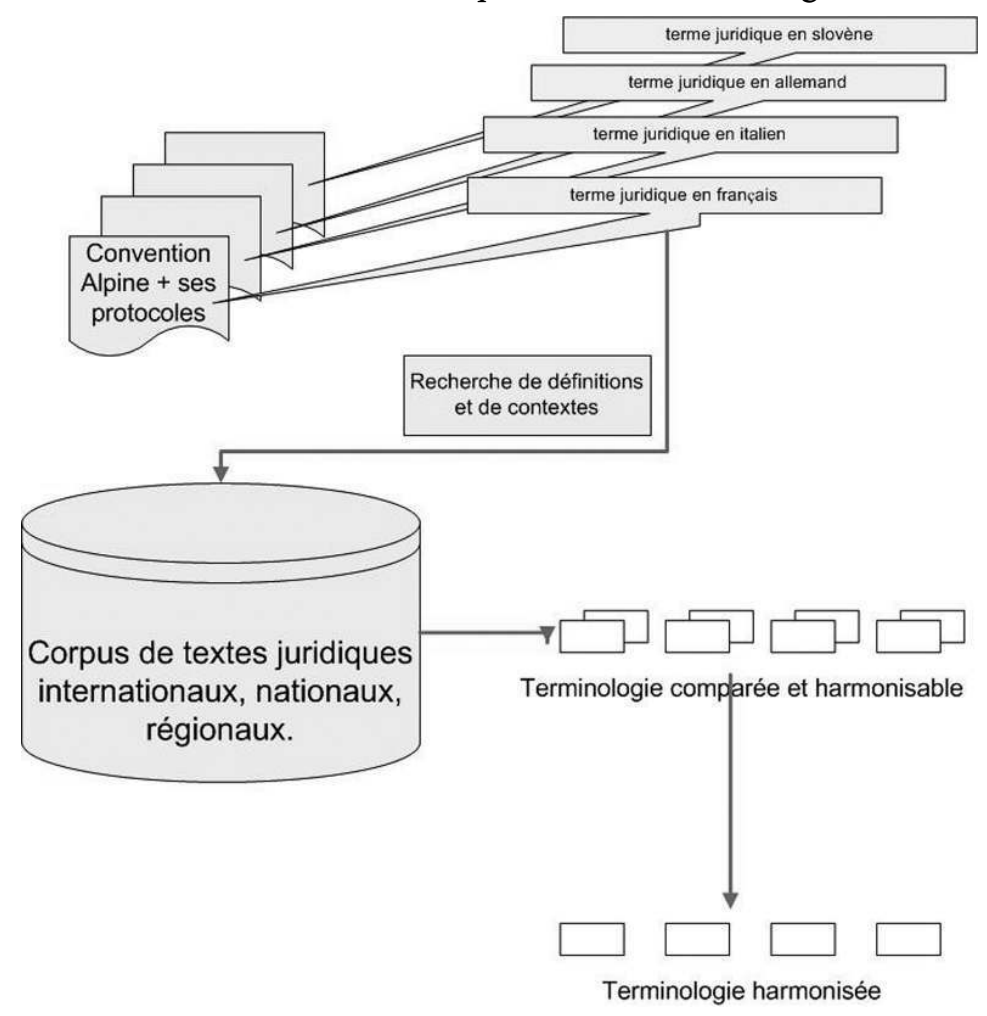

Figure : Le corpus, élément central du travail d'harmonisation

7 Cette liste de termes «correspondants» dans les quatre langues devient l'objet d'une description et d'une comparaison d'un point de vue juridique. C'est pourquoi nous avons 
rassemblé un corpus de textes juridiques en matière d'environnement et d'aménagement du territoire intéressant l'arc alpin. La collection contient des normes internationales et européennes, nationales et régionales des États Parties à la Convention. Les partenaires du projet ont réuni les textes intéressant les domaines de l'aménagement du territoire et du développement durable de six systèmes juridiques nationaux (l'Allemagne, l'Autriche, la France, l'Italie, la Slovénie et la Suisse), des systèmes juridiques européen et international ainsi que les textes directement liés au système conventionnel alpin. Ils ont été sélectionnés par des juristes à partir d'un ensemble de critères élaborés pour le projet. Seuls des documents intégraux (pas d'extraits ni de paragraphes isolés) ont été utilisés (< www.eurac.edu/lexalp>). L'article de E. Chiochetti et V. Lyding (2006) présente et justifie cette approche de manière détaillée. Le corpus compte aujourd'hui près de 19 millions de mots issus de 3095 différentes sources primaires de droit et se présente déjà comme une ressource pour améliorer la communication transfrontalière juridique.

Malgré l'étendue de ce corpus, il n'était pas suffisant pour décrire tous les concepts. Le problème s'est posé surtout pour les définitions légales, qui n'ont augmenté que récemment dans la législation. Nous avons donc fait des références à d'autres documents, recensés dans une base de données bibliographiques. Nous avons ainsi pu pallier le fait que depuis la création du corpus de nouveaux textes juridiques ont été adoptés ou que des textes du corpus ont été amendés. Ainsi, il est possible d'avoir un accès direct à la loi française adoptée en 2006 sur les parcs nationaux et régionaux ${ }^{8}$ bien qu'elle n'ait pas été intégrée au corpus étant donné son adoption postérieure à la collecte des normes.

De même, le fait de pouvoir accéder aisément aux références permet aussi d'être «en règle » par rapport aux dispositions réglementant le droit d'auteur ${ }^{9}$ car des ouvrages juridiques ont souvent été utilisés pour l'élaboration de notes explicatives et l'extraction de définitions ou de contextes. Un système clair d'abréviations permet d'identifier aisément les documents utilisés et de pouvoir les consulter si nécessaire. À partir de cette description de la terminologie de la Convention alpine et sur le fond de la terminologie juridique dans les systèmes juridiques connexes, nous arrivons à une terminologie harmonisable.

Il est nécessaire de souligner que si les termes ne semblent pas toujours équivalents, les traités internationaux que sont la Convention alpine et ses protocoles d'application sont à considérer comme harmonisés. La Conférence alpine a en effet constaté en 2000 que « les protocoles [...] ont été entièrement harmonisés sur les plans linguistiques et stylistiques, et ce sans qu'aucune modification de fond n'ait été apportée $\aleph^{10}$. Cela était d'ailleurs déjà le cas avant l'an 2000 en vertu de l'article 33, paragraphe 1, de la Convention de Vienne sur le droit des Traités qui énonce que " [lorsqu'] un traité a été authentifié en deux ou plusieurs langues, son texte fait foi dans chacune de ces langues, à moins que le traité ne dispose ou que les parties ne conviennent qu'en cas de divergence un texte déterminé l'emportera ».Il est précisé au paragraphe 3 de ce même article que «[les] termes d'un traité sont présumés avoir le même sens dans les divers textes authentiques ».

Trois interprétations de ce paragraphe 3 sont envisageables : a) les textes sont équivalents au moment de l'application du paragraphe 3 et la norme protège cette équivalence contre une interprétation divergente dans l'avenir ; b) les textes n'étaient pas équivalents avant l'application du paragraphe 3 , mais ils le sont après. Le paragraphe 3 fonctionne comme une règle d'interprétation qui limite les textes au seul sens qui est soutenu par toutes les langues: l'interprétation d'un texte est limitée par les limites d'interprétation des autres ; c) les textes n'étaient pas équivalents avant, et ils ne le sont 
pas après - ils sont seulement « présumés » équivalents par une fiction juridique. Dans les trois cas, les auteurs du paragraphe 3 reconnaissent que le parallélisme linguistique risque de biaiser la précision du contenu - un effet qui peut être contrebalancé par l'harmonisation. La solution n'intervient plus au niveau du texte, mais au niveau des termes : lorsque la terminologie correspond, le contenu correspond lui aussi.

Ensuite, la terminologie harmonisable est proposée à une commission technique, le "groupe d'harmonisation", qui décide quelle est la terminologie devant être considérée comme effectivement harmonisée ${ }^{11}$. Le groupe d'harmonisation a aussi, si nécessaire, proposé de nouveaux termes (parfois de provenance d'autres systèmes juridiques) afin qu'ils soient utilisés par la Convention alpine. Cette terminologie nouvelle n'est évidemment pas intégrée dans les traités, mais elle est proposée pour de futures traductions. Ce type d'intervention créative a été nécessaire, parce que les termes de la Convention alpine sont parfois flous et peu adaptés. Nous pouvons alors constater une interprétation plus libre et large du texte de la Convention, inspirée des différents systèmes juridiques décrits dans le projet. En pratique, il était beaucoup plus facile d'adopter des termes et définitions issus de textes juridiques européens car le droit de ce système multilingue correspond et cela facilite la tâche qui est d'aboutir à une correspondance normative et linguistique. Cependant, il faut aussi souligner que cette facilité doit être relativisée car les définitions peuvent être différentes pour un même système juridique selon les différentes branches du droit ${ }^{12}$, voire selon les différents textes. Ainsi la définition d'activité agricole et d'agriculteur en droit européen peut être plus ou moins large selon les textes. Mais l'extension du champ d'application de ces concepts que sont agriculteur et activité agricole est de plus en plus répandue dans la mesure où la politique agricole commune "incline davantage vers un soutien des activités environnementales et de diversification par les services exercées en milieu rural, que vers un soutien de la production agricole comme elle le faisait initialement » (BarabéBouchard et Hérail, 2007 : 13).

La définition d'un terme conditionne l'application ultérieure du texte, même si le texte de la Convention alpine et de ses protocoles d'application ne change pas ${ }^{13}$. Ceci pose des questions de légitimité, parce que les harmonisateurs semblent changer la portée d'une norme. Nous ne pouvons objecter que l'harmonisation dans le projet n'est autre que l'activité de chaque interprète du texte : rendre compatible les quatre sens divers en un sens commun. Le projet ne décide pas délibérément de ce sens commun, mais documente le résultat d'un processus difficile pour aider les traducteurs, mais aussi, à terme, le juge. De toute manière, il convient de regarder d'un peu plus près les implications de l'harmonisation juridique.

\section{L'harmonisation : une tâche aux multiples facettes}

L'harmonisation est une tâche complexe avec de multiples relations telles que la description-prescription (a), l'extraction-définition d'un sens (b) et enfin la réceptionnormalisation (c), car définir un terme juridique implique des conséquences en termes de normalisation. 


\subsection{Décrire en vue de prescrire}

Les termes sont décrits afin d'être appréhendés d'une manière commune et sont alors générateurs d'obligations. L'interprétation « n'est [donc] pas un exercice abstrait, d'ordre intellectuel, qui dégagerait en soi le sens de la règle " (Combacau et Sur, $2006: 168)$. Il s'agit d'une "activité positive, comportant un objet particulier et un but pratique» (Porta, 2007 : 723). L'objectif est ici une appréhension et une application uniformes des dispositions du système conventionnel alpin. Ainsi, l'appréhension d'un terme peut être envisagée de différentes façons, ce qui aura des conséquences juridiques différentes. C'est pourquoi les représentants des États Parties, qu'ils soient observateurs ou partenaires directs du projet LexALP, se montrent attentifs aux définitions et donc à l'interprétation des termes, qui est donnée à travers ce projet $^{14}$. Ceci relève des mêmes préoccupations que pour le droit communautaire et son interprétation de manière uniforme pour tous les États membres. Pour reprendre les termes de J. Porta, « [la] convergence des législations ne serait qu'un leurre, si le droit harmonisé avait, dans chaque ordre juridique national, des interprétations et des applications divergentes » (Porta, 2007 : 273).

Le terme transfrontalier illustre l'importance du signifiant donnée aux termes (Randier, 2008). Le groupe d'harmonisation avait le choix entre une définition large (et innovante) et une définition stricte (et classique). La représentante de la Délégation Générale à la langue française et aux langues de France ${ }^{15}$, observatrice du projet LexALP, s'opposait à la première acception large et innovante (évolutive) du terme, car elle ne correspondait pas à l'appréhension commune du terme dans la langue française. Selon la définition large et évolutive, issue d'un rapport du Réseau alpin des espaces protégés, instrument de la Convention alpine, transfrontalier « ne signifie pas [...] seulement de part et d'autre des frontières nationales, mais cela concerne également [...] les frontières nationales administratives» (Réseau Alpin des Espaces Protégés , 2004: 30). Or selon l'acception française juridique, identique à l'acception commune, transfrontalier signifie de part et d'autre des frontières nationales. La frontière s'entend selon le Dictionnaire juridique de G. Cornu comme la «[1]igne séparant le territoire de deux États» (Cornu, 2007 : 431). La position de la DGLFLF a donc été respectée et le groupe d'harmonisation a finalement adopté la définition classique du terme. Cela répond de près aux exigences de la Convention de Vienne sur le droit des traités qui énonce à l'article 31, paragraphe 1, qu'« [un] traité doit être interprété de bonne foi suivant le sens ordinaire à attribuer aux termes du traité dans leur contexte et à la lumière de son objet et de son but ». Le paragraphe 4 de ce même article précise qu'un «terme sera entendu dans un sens particulier s'il est établi que telle était l'intention des parties ». Il aurait été intéressant ici de pouvoir consulter les travaux préparatoires de ce protocole. De plus, il semble un cas typique dans le domaine de l'environnement où « une interprétation tenant compte de l'évolution des choses doit être préférée lorsqu'elle est de nature à permettre une application effective du traité »(Canal-Forgues et Rambaud, $2007: 41$ ).

15 En général, la conflictualité " politique » lors des séances du groupe d'harmonisation était assez atténuée à cause de la nature pré-politique des décisions. En fait, les termes harmonisés apparaissent un peu partout dans les textes. C'est pourquoi il est pratiquement impossible d'en prévoir les conséquences sur la législation de fond et sur les traités présents et futurs. En effet, il suffirait d'insérer une négation dans la norme pour changer complètement l'effet juridique ${ }^{16}$. Dans le cas d'" espace transfrontalier », le contexte et les conséquences pratiques étaient très clairs. La France se définit dans sa 
Constitution comme "une et indivisible», et ne peut donc pas accepter d'avoir des frontières internes à son territoire. "Transfrontalier » concerne alors seulement les relations entre des États souverains.

L'appréhension juridique d'un terme diffère parfois de son appréhension commune, la dernière n'ayant pas pour objet de délimiter le champ d'obligations particulières. Ainsi le terme espèce peut être appréhendé différemment dans des textes scientifiques ou juridiques (voir aussi Amirante (2007)). La définition scientifique est précise et très claire tandis que la définition juridique se veut plus globalisante afin d'aboutir à une protection des espèces la plus efficace possible. Ainsi l'espèce est définie communément comme un " ensemble d'individus animaux ou végétaux semblables par leur aspect, leur habitat, féconds entre eux mais ordinairement stériles avec tout individu d'une autre espèce » ( Dictionnaire Général, 2006) tandis qu'elle est définie à l'article 1a) de la "Convention sur le commerce international des espèces de faune et de flore sauvages menacées d'extinction $»^{17}$ comme "[t]oute espèce, sous-espèce, ou une de leurs populations géographiquement isolée ${ }^{18}$. Le caractère tautologique de cette dernière définition proposée au groupe d'harmonisation et refusée par les linguistes du projet LexALP, est issu de l'objectif du traité qui est d'aboutir à la protection la plus large possible des espèces-objets du traité en protégeant aussi bien les espèces que les sous-espèces de celles-ci. Le groupe d'harmonisation décida finalement d'adopter la définition commune et scientifique de la notion d'espèce ${ }^{19}$.

17 La prescription suit la description. Alors que la description traite d'un objet déterminé, la prescription s'applique à des classes d'objets réels et potentiels, présents et futurs. Faire une description est plus facile que faire une prescription, car une norme peut s'appliquer à beaucoup d'objets à venir et qui n'étaient pas prévus par l'auteur de la norme.

Un exemple illustrant ce besoin de clarté se trouve dans la définition de la notion même d'environnement. Celle-ci peut être problématique car elle conditionne des obligations comme par exemple celle des études d'impact sur l'environnement. La notion d'environnement peut être alors définie comme l'objet sur lequel s'applique l'étude d'impact. Ainsi la directive 85/335/ CEE envisage dans son article 3 l'environnement comme «- l'homme, la faune et la flore, - le sol, l'eau, l'air, le climat et le paysage, l'interaction entre les facteurs visés aux premier et deuxième tirets, - les biens matériels et le patrimoine culturel ». Cette définition a été conçue de manière très large afin d'envisager ses différents usages.

19 Le terme "élément digne de protection $»^{20}$ peut illustrer ce lien entre description et prescription et la nécessité d'avoir une interprétation claire du terme afin de savoir dans quelle mesure s'applique la prescription issue de l'article $12 \mathrm{du}$ protocole Protection de la nature et Entretien des paysages de la Convention alpine. En effet, selon cet article « [les] Parties contractantes prennent les mesures adéquates pour établir un réseau national et transfrontalier d'espaces protégés, de biotopes et d'autres éléments protégés ou dignes de protection dont le caractère est reconnu ». Une définition a été extraite indirectement de l'article 2 du protocole Transports : "Élément du patrimoine naturel et culturel qui, du fait de sa valeur, devrait être soumis à un régime spécial de protection et de gestion pour atteindre des objectifs spécifiques de qualité. »Ce terme, présent dans la législation suisse de protection de la nature, illustre aussi le va-et-vient entre les droits nationaux et le droit international ${ }^{21}$.

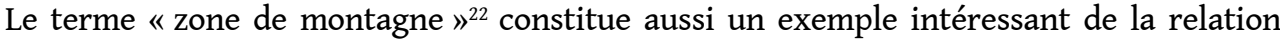
entre la définition et la génération d'obligations. En effet, le contenu de ce terme est 
important car il génère des prescriptions, notamment en matière d'agriculture de montagne. Le groupe d'harmonisation ne pouvait accepter la définition proposée initialement car elle était fondée sur des critères géographiques très précis alors que ce sont les États Parties qui sont compétents pour établir de tels critères. Le groupe d'harmonisation a finalement opté pour une définition issue de l'article 18,1 du règlement n 1257/1999, qui ne s'appuie pas sur de tels critères : «zone [...] caractérisée par une limitation considérable des possibilités d'utilisation des terres et un accroissement sensible des couts des travaux en raison de : soit l'existence de conditions climatiques très difficiles en raison de l'altitude, se traduisant par une période de végétation sensiblement raccourcie, soit la présence, à une altitude moindre, de fortes pentes dans la majeure partie du territoire, telles que la mécanisation ne soit pas possible ou bien nécessite l'utilisation d'un matériel particulier très onéreux, soit la combinaison de ces deux facteurs lorsque l'importance du handicap résultant de chacun d'eux pris séparément est moins accentuée, à condition que de cette combinaison résulte un handicap équivalent. » Une définition fondée sur des critères géographiques précis tels que l'altitude n'existe d'ailleurs pas et ne peut pas exister dans les textes communautaires (Schuler, Stucki, Roque et Perlik, 2004).

\subsection{Extraire afin de donner du sens}

La description des termes peut aussi permettre de donner du sens à des concepts issus d'un système juridique national particulier et qui n'apparaissent pas en tant que tels dans d'autres. Cette situation a eu lieu lors de ce travail d'harmonisation car, chaque protocole ayant été rédigé par un groupe de travail dirigé par un État Partie, certains concepts sont directement hérités du système juridique de cet État. Ainsi, certains concepts du protocole « Protection de la nature et entretien des paysages ", rédigé par un groupe de travail allemand, sont issus du système juridique allemand et ne se retrouvent pas dans les autres systèmes juridiques nationaux. Décrire les termes par l'élaboration d'une définition permet d'attacher un sens à ces termes et de les rendre appréhendables par les juristes des autres États Parties, voire de rattacher ce concept à des concepts existant dans leur droit national, mais portant une appellation différente.

21 Nous pouvons ici prendre l'exemple zone de protection du paysage. La description du terme n'a pas permis de trouver un équivalent exact, mais il a alors été possible de le rapprocher de concepts existant en droit national. Les zones de protection du paysage n'existent pas en tant que catégorie d'espace protégé en droit français mais avec la description du terme un lien a pu être fait avec la procédure des directives de protection et de mise en valeur des paysages qui, selon l'article L. 350-2 du code de l'environnement à lire en relation avec l'article L. 642-1, vise la désignation de zones de protection du patrimoine architectural, urbain et paysager (« espaces à protéger ou à mettre en valeur pour des motifs d'ordre esthétique, historique ou culturel »). Ces différences de catégorisation des espaces protégés à travers l'arc alpin sont issues des différentes politiques en matière de protection de la nature. Cela a pu être noté à plusieurs reprises (Veyret, 2002) et est une illustration, à échelle plus réduite, de ce qui a pu être constaté au niveau international (IUCN, 2002). 


\subsection{Réception et normalisation}

Le travail d'harmonisation du projet LexALP représente un « va-et-vient » entre les textes du système conventionnel alpin et les textes nationaux, européens et internationaux. En effet, nous partons des termes de la Convention alpine - souvent issus eux-mêmes des textes nationaux -, que nous décrivons avec des textes des autres systèmes juridiques et des définitions que nous pouvons extraire de ces textes. Le groupe d'harmonisation s'inspire généralement des textes juridiques internationaux et européens pour donner un sens aux termes de la Convention alpine. Ainsi, les termes de la Convention alpine deviennent plus précis, mais cela peut alors révéler des inconsistances dans le texte de la Convention alpine ${ }^{23}$. Lors de la conclusion du traité, de telles inconsistances pouvaient seulement être soupçonnées, et c'est pour les éviter qu'a été adoptée la formule "textes harmonisés » (voir supra). Maintenant, après une comparaison juridique méticuleuse des concepts derrière chaque expression, les inconsistances sont révélées et l'on peut chercher à y remédier en adoptant ensemble une définition commune. Ici il faut souligner que formellement, l'harmonisation ne se réfère qu'aux dénominations, mais en pratique la définition est non seulement la justification de la correspondance, mais donne aussi le champ d'application de la norme linguistique : le terme n'est prescrit que lorsqu'il y a la volonté d'exprimer le concept de fond - qui se trouve justement dans la définition commune.

Le droit international s'érige sur des droits nationaux, des concepts, des méthodes et des fonctionnements. Ainsi est née la Convention alpine. Maintenant la Convention alpine fait naitre, après inspiration d'un système national ou international, des concepts qui sont pour la plupart des États membres de nouveaux concepts. Nous pouvons constater que le schéma traditionnel d'approvisionnement terminologique et conceptuel de bas en haut fonctionne aussi en inverse. Cela peut être une inspiration pour les systèmes nationaux, et le cercle vertueux d'innovation peut continuer. Ce procédé en cercles pourrait mener à une harmonisation itérative, et avec chaque cycle les concepts se rapprochent. Mais, en droit européen, le principe de subsidiarité garantit que les innovations propres des États constituent un élément créatif suffisamment fort pour ne pas unifier silencieusement le droit européen.

\section{Portée juridique du projet LexALP}

De quelle nature juridique sont les résultats du projet LexALP? L'harmonisation des termes, c'est-à-dire la recherche d'une équivalence des termes dans les quatre langues de la Convention alpine, exige d'en donner des définitions et donc de les interpréter. Selon le Dictionnaire juridique de G. Cornu, l'interprétation est une "opération qui concerne à discerner le véritable sens d'un texte obscur " et cela "désigne aussi bien les éclaircissements donnés par l'auteur même de l'acte (loi interprétative, jugement interprétatif), que le travail d'un interprète étranger à l'acte (interprétation doctrinale, interprétation judiciaire d'une convention, interprétation ministérielle d'une loi)» (Cornu, 2007: 510). Les harmonisateurs de LexALP appartiennent à cette seconde catégorie d'« interprètes étrangers à l'acte » car ils n'ont pas été les auteurs de ces traités de droit international que sont la Convention alpine et ses protocoles d'application. Il s'agit alors d'une interprétation concertée car il y a l'«adhésion des membres à l'interprétation commune d'une règle visée »(Combacau et Sur, 2007: 175). Cette 
démarche est originale car "l'une des caractéristiques juridiques de cet ordre juridique tient précisément au fait que chaque sujet y détient la compétence, généralement discrétionnaire, d'interpréter pour lui-même le sens et la portée des droits et des obligations qu'il possède en vertu de normes internationales, coutumes et principes généraux du droit, aussi bien que traités » (Dupuy, 2006 : 322).

Les définitions fournies dans le cadre du projet LexALP n'appartiennent pas au corps du traité et ne sont donc pas juridiquement contraignantes. À l'inverse, les définitions issues de l'article 2 du Protocole Transports, du fait de leur insertion dans le traité, ont un caractère directement contraignant. Mais le fait que les définitions soient approuvées par des représentants des gouvernements des États alpins et par le Secrétariat permanent de la Convention alpine leur confère une valeur de recommandation, c'est-à-dire une « invitation à agir dans un sens déterminé » (Cornu, 2007: 771). L'interprétation des termes pourrait donc être considérée comme de la soft law («droit international flexible») (Carreau, 2007: 194), type de droit qui «s'intègre dans tout un processus d'assouplissement de l'élaboration du droit international » (Combacau et Sur, 2007 : 173). Ces interprétations que sont les définitions constituent des indications devant influencer les acteurs du système conventionnel alpin, y compris les acteurs non étatiques.

Le travail d'harmonisation des termes de la Convention alpine est particulier car il va plus loin que celui du juge, qui se tient normalement strictement au texte en cause dans une affaire. L'interprète harmonisateur va aussi plus loin que le juge car il ne peut pas se contenter des définitions contenues dans le système conventionnel alpin, qui sont, comme nous venons de le rappeler, très peu nombreuses.

Finalement, les juges nationaux qui seront les prochains interprètes du texte de la Convention décideront de la valeur de la terminologie harmonisée. Ce sera une procédure probablement non uniforme, parce que la jurisprudence qui concerne l'interprétation de dispositions du système conventionnel alpin a essentiellement un caractère national. La jurisprudence relative à la Convention alpine commence d'ailleurs à s'étoffer en Autriche (Cuypers, 2007; Alpenkonvention, 2007). L'interprétation des juges des États Parties est une interprétation unilatérale et "constitue une assertion unilatérale qui ne lie pas en elle-même les autres sujets » (Combacau et Sur, 2006 : 173); elle est donc sans contrainte pour les autres États contractants. Mais il sera alors intéressant de voir si, dans le futur, les approches des différents États membres se rejoignent et de quelle manière elles s'influenceront mutuellement. Le juge communautaire pourrait être amené à se prononcer dans le cadre d'un tel processus car l'Union européenne a ratifié la Convention alpine et certains de ses protocoles ${ }^{24}$.

De même, il sera intéressant d'examiner quelle est l'influence de la terminologie harmonisée sur la jurisprudence nationale : est-ce que le juge acceptera le sens harmonisé comme s'il n'y avait jamais eu d'écart interprétatif entre les versions linguistiques? Estce que sera discutée la question de savoir si et dans quelle mesure la terminologie harmonisée constitue une source de droit valable, légitime car acceptée par les représentants du Secrétariat permanent de la Convention alpine? La terminologie harmonisée se développe discrètement mais elle a la capacité de changer la perception du droit issu du système conventionnel alpin et forme ainsi de la vraie soft law. 


\section{BIBLIOGRAPHIE}

AMIRANTE D. (2007) : L'autonomie scientifique du droit de l'environnement, Réflexions de droit comparé, dans M. Prieur, Pour un droit commun de l'environnement, Paris, Dalloz, p. 3-19.

BARABÉ-BOUCHARD V. et HÉRAIL M. (2007) : Droit rural, Paris, Ellipses.

BERTHELOT P. (2008) : La standardisation dans les actes législatifs de l'Union européenne et les bases de terminologie, Séminaire sur la normalisation, l'harmonisation et la planification linguistique, Bolzano/Bozen, 8 février 2008.

CANAL-FORGUES E. et RAMBAUD P. (2007) :Droit international public, Paris, Flammarion (dir.) (Collection Champs Université).

CARREAU D. (2007) :Droit international public, Paris, Pedone Éd. (Collection Études internationales).

сhiocchetti e. et voltmer l. (dir.) (2008a) :LexAlP - Harmonising Alpine Terminology, dans chiocchetti E. et voltmer L. (dir.), Harmonising Legal Terminology, Bolzano, EURAC, p. 45-55.

CHIOCCHETTI E. et VOLTMER L. (2008b) : Normalisation, harmonisation et planification linguistique, dans E. Chiocchetti et L. Voltmer(dir.) (2008),Harmonising Legal Terminology, Bolzano/Bozen, EURAC.

сніоснетті е. et uyding v. (2006) : Multilingual Corpus for Terminology Work: The LexALP Corpus of Legal Texts, dans A. ABEL, M. STUFLESSER et M. PUTZ, Mehrsprachigkeit in Europa, Tagungsband, Bolzano/Bozen, EURA Research, p. 505-513.

COMBACAU J. et SUR S. (2007), Droit international public, Paris, Domat droit public, Montchrestien, $7^{\mathrm{e}}$ édition.

CORNU G. (dir.) (2007) : Vocabulaire juridique, Paris, PUF.

cuYpers s. (2007) : Die Umsetzung der Alpenkonvention und ihrer Durchführungsprotokolle im österreichischen Recht unter vergleichender Darstellung einzelner Aspekte der Rechtslage der Europäischen Gemeinschaft und das deutschen Recht, Dissertation, Rechtswissenschaft, Innsbruck.

DEL'ANNA M.V., SERPENTIN C., gUALDO R. (2008) : Résultats du projet de recherche « Il regime linguistico dell'amministrazione europea », « Note linguistiche e lessicali su testi giuridici di uffici amministrativi comunitari », actes du séminaire « La ricerca nella comunicazione linguistica: Modelli teorici e a.

DELORME H. (dir.) (2004) : La politique agricole commune, Anatomie d'une transformation, Presses de Sciences Po. 
Dictionnaire général (2006) : Le Petit Larousse Illustré 2007, Paris, Larousse. DUPUY P. M. (2006) :Droit international public, 8 e édition, Paris, Précis Dalloz. HEUTGER V. (2004) : European Integration online Papers (EIoP), vol.7, $\mathrm{n}^{\circ} 2 ;<\mathrm{http}$ //eiop.or.at/eiop/ texte/2004-002a.htm> (page consultée le 18/02/2008).

ISO 860:1996(F), Norme de l'International Standard Office, section 6.2.1.2.

ISO 860:1996(F): Norme de l'International Standard Office, section 3.1.

IUCN (1994) : Guidelines for Protected Areas Management Categories, IUCN, Cambridge/ Gland.

IUCN/ UNEP(2002) : Speaking a common Language, Uses and performance of the IUCN System of Management Categories for Protected Areas, Cardiff University.

LEBENSMINISTERIUM ÖSTERREICH UND PERMANENTES SEKRETARIAT DER ALPENKONVENTION (2007) :

Die Alpenkonvention: Handbuch für ihre Umsetzung, Rahmenbedingungen, Leitlinien und Vorschläge für die Praxis zur rechtlichen Umsetzung der Alpenkonvention und ihrer Durchführungsprotokolle, Klagenfurt.

OLIVIER J. (2005) : L'Union mondiale pour la nature (UICN), Une organisation singulière au service de l'environnement, Collection Les Travaux du CERIC, Bruylant(dir.), Bruxelles, p.192.

PORTA J. (2007) : La réalisation du droit communautaire, Essai sur le gouvernement juridique de la diversité, LGDJ, Paris, p.723.

RANDIER C. (2008) : Definitions for Harmonising Legal

Terminology, Examples from the Protocol on the

Conservation of Nature and Countryside, dans E. Chiocchetti et L. Voltmer (dir.) (2008),Harmonising Legal Terminology, Bolzano/Bozen, EURAC, p.91-105.

Rapport final de la Sixième Conférence alpine des 30 et 31 octobre 2000 à Luzerne. RÉSEAU ALPIN DES ESPACES PROTÉGÉS (2004) : Typologie des espaces protégés alpins - Cadre juridique et statuts de protection, Gap, Collection Les dossiers du Réseau alpin, $n^{\circ} 8$.

SCHULER M., STUCKI E.W., ROQUE O., PERLIK M. (2004) : Mountain Areas in Europe: Analysis of mountain areas in EU members states, acceding and other European countries. Final Report, European Commission Contract $\mathrm{n}^{\circ}$ 2002.CE.16.0.AT.136. NORDREGIO - Nordic Centre for Spatial Development.

SECRÉTARIAT PERMANENT DE LA CONVENTION ALPINE ET RÉSEAU ALPIN DES ESPACES PROTÉGÉS (dir.) (2004) : Espaces protégés transfrontaliers et réseau écologique dans les Alpes, Étude sur mandat de la Convention alpine, Signaux alpins n 3, Gap, p. 30.

VEYRET Y. (2002) : Les montagnes, discours et enjeux géographiques, Paris, SEDES (Collection Dossiers des Images Économiques du Monde).

voltmer l. (2008) : Experiences in Harmonising, dans E. chiocchettiet L. Voltmer (dir.) (2008),Harmonising Legal Terminology, Bolzano/Bozen, EURAC, p. 69-90. 
WEBER-LEJEUNE S. (1997): Legaldefinitionen unter besonderer Berücksichtigung des Umweltrechts, Berlin, Berlin-Verlag Spitz.

\section{NOTES}

1. <www.eurac.edu/lexalp>

2. Normalisation : «Intervention linguistique d'un organisme autorisé visant à fixer l'usage d'un terme ou d'un ensemble de termes en privilégiant ou en déconseillant l'emploi par une communauté ciblée. » Bureau de la traduction du Canada, <http://www.translationbureau.gc.ca/ index.php?cont=700\&lang=francais $>$ : 08.10.2008. Par ailleurs, la norme ISO 860:1996(F) définit ainsi l'harmonisation : 3.1 harmonisation des notions : réduction ou élimination des différences mineures entre deux notions très semblables ou plus. NOTE $1:$ L'harmonisation des notions fait partie intégrante de la normalisation. 3.2 harmonisation des termes : activité devant aboutir à la désignation, dans plusieurs langues, d'une même notion par des termes qui reflètent les mêmes caractères ou des caractères similaires dont la forme est la même ou légèrement différente (Chiocchetti et Voltmer, 2008).

3. La norme ISO 860:1996(F), 6.2.1.2, en est un exemple.

4. <http://iate.europa.eu/>

5. Cf. les résultats du projet de recherche « Il regime linguistico dell'amministrazione europea », Maria Vittoria Dell'Anna, Carla Serpentini, Riccardo Gualdo, « Note linguistiche e lessicali su testi giuridici di uffici amministrativi comunitari», actes du séminaire «La ricerca nella comunicazione linguistica: Modelli teorici e metodologici » du 1-2 février 2008 à l'Université Macerata.

6. Cf. le projet de recherche AUTOTRAD de l'Université de Barcelone, <http://www.fti.uab.es/ autotrad> (page consultée le 29/01/2008).

7. Il s'agissait de l'association INTRALP.

8. Loi $n^{\circ} 2006-436 \mathrm{du} 14$ avril 2006 relative aux parcs nationaux, aux parcs naturels marins et aux parcs naturels régionaux (JORF du 15 avril 2006, p. 1 et suivantes).

9. Voir notamment pour les dispositions de droit français l'article L. 122-5 du Code la propriété intellectuelle.

10. Rapport final de la Sixième Conférence alpine des 30 et 31 octobre 2000 à Luzerne, point 5.6 $\mathrm{du}$ procès-verbal. Les protocoles harmonisés sont «Aménagement du territoire et développement durable », " Agriculture de montagne », "Protection de la nature et entretien des paysages ", « Forêts de montagne », « Tourisme », « Protection des sols et énergie ».

11. Sur la composition et les tâches du groupe d'harmonisation, voir E. Chiocchetti et L. Voltmer (2008a).

12. Ainsi par exemple, la notion de «travailleur » sera interprétée différemment selon qu'on se situe en droit du travail ou en droit des affaires (Berteloot, 2008).

13. Une procédure spécifique pour émettre des amendements à la Convention alpine est prévue par l'article 10 de la Convention alpine.

14. Il est à noter que le Protocole "Règlement des différends de la Convention alpine " prévoit une procédure spécifique en cas de différend entre les parties contractantes relatif à l'interprétation ou à l'application de la Convention alpine ou de l'un de ses protocoles.

15. Délégation générale à la langue française et aux langues de France, (Ministère de la Culture et de la Communication), membre du groupe d'harmonisation, ci-après DGLFLF.

16. Il y a ici un parallèle à l'idée de Rawls: la prise de décisions dans la position originelle derrière un « voile de l'ignorance ». 
17. Convention sur le commerce international des espèces de faune et de flore sauvages menacées d'extinction, signée à Washington le 3 mars 1973 et amendée à Bonn, le 22 juin 1979.

18. Et il faut souligner que les accords internationaux se réfèrent généralement à une liste d'espèces protégées qu'ils annexent alors à l'accord afin de délimiter le champ d'application de celui-ci.

19. Sur le conflit entre les définitions scientifiques et les définitions légales dans le domaine de la protection de l'environnement, voir Weber-Lejeune, 1997.

20. Beni ambientali meritevoli di protezione/ élement digne de protection/zavarovanja vredni deli okolja/ schützenswertes Objekt.

21. L'article 18, 1ter, énonce que « [si], tous intérêts pris en compte, il est impossible d'éviter des atteintes d'ordre technique aux biotopes dignes de protection, l'auteur de l'atteinte doit veiller à prendre des mesures particulières pour en assurer la meilleure protection possible, la reconstitution ou, à défaut, le remplacement adéquat ». L'article 18, paragraphe 2, énonce que «dans la lutte contre les ravageurs, notamment dans la lutte au moyen de substances toxiques, il faut éviter de mettre en danger des espèces animales et végétales dignes de protection».

22. Berggebiet/ zona montana/ gorsko območje.

23. Ainsi, nous avons pu noter une différence dans le texte du protocole sur la protection de la nature concernant le terme atteinte à la nature et aux paysages. Tandis que le terme allemand Eingriff in Natur und Landschaft correspond, les termes italien et slovène (intervento nella natura e nel paesaggio, poseg $v$ naravo in krajino) ne correspondent pas : en français et en allemand, le terme se rapporte à une action aux conséquences négatives, tandis que cela est neutre en italien et en slovène.

24. Dans le respect des conditions fixées par l'article $300 \mathrm{TCE}$, les accords conclus par la Communauté avec des États tiers ou des organisations internationales constituent une source à part entière du droit communautaire.

\section{RÉSUMÉS}

Dans cet article, nous présentons un point de vue sur le projet INTERREG LexALP, relatif à l'harmonisation de la terminologie juridique dans les quatre langues de la Convention alpine (italien, français, slovène, allemand). Nous y démontrons la nécessité et l'utilité de l'harmonisation juridique, et expliquons le procédé suivi ainsi que les outils utilisés. Cette activité a requis le développement d'une méthodologie nouvelle et soulève des problèmes intéressants de légitimation politique, de théorie du droit et de philosophie des langues. Notre démonstration est agrémentée d'exemples de termes harmonisés pour illustrer ces problèmes.

In this article, we present a point of view on the INTERREG project LexALP, related to legal terminology harmonisation in four alpine languages (Italian, French, Slovene, German). We show that legal harmonisation is useful, and even necessary, and we present the procedure followed and the tools used. The harmonising of legal terms has lead to a new methodology and to challenging new problems in the fields of political legitimation, legal theory and the philosophy of language. Our demonstration is accompanied by examples of harmonised terms to explain the implications. 
AUTEURS

LEONHARD VOLTMER

European Academy, Bolzano, Italie 